\title{
Statistical study of medium-scale traveling ionospheric disturbances observed with the GPS networks in Southern California
}

\author{
Nobuki Kotake ${ }^{1}$, Yuichi Otsuka ${ }^{1}$, Tadahiko Ogawa ${ }^{1}$, Takuya Tsugawa ${ }^{2}$, and Akinori Saito ${ }^{3}$ \\ ${ }^{1}$ Solar-Terrestrial Environment Laboratory, Nagoya University, Toyokawa, Aichi 442-8507, Japan \\ ${ }^{2}$ MIT Haystack Observatory, Atmospheric Sciences Off Route 40, Westford, MA 01886-1299, U.S.A. \\ ${ }^{3}$ Graduate School of Science, Kyoto University, Kitashirakawa-Oiwakechou, Sakyou-ku, Kyoto 606-8502, Japan
}

(Received June 12, 2006; Revised August 18, 2006; Accepted September 4, 2006; Online published March 21, 2007)

\begin{abstract}
Using global positioning system (GPS) data taken from 350 dual-frequency GPS receivers in Southern California in 2002, we investigated two-dimensional maps of total electron content (TEC) perturbations with a time resolution of $30 \mathrm{~s}$ and a spatial resolution of $0.15^{\circ} \times 0.15^{\circ}$ in longitude and latitude to reveal statistical characteristics of medium-scale traveling ionospheric disturbances (MSTIDs). We found that MSTIDs can be categorized into three types. One type is daytime MSTIDs, which frequently occur in winter and equinoxes. Since most of the daytime MSTIDs propagated southeastward, we speculate that the daytime MSTIDs could be caused by atmospheric gravity waves in the thermosphere. A second type is nighttime MSTIDs, which frequently occur in summer. Nighttime MSTIDs propagate southwestward. This propagation direction is consistent with the idea that polarization electric fields could play an important role in generating nighttime MSTIDs. The third is dusk MSTIDs, which frequently occur in summer and propagate northwestward. Dusk MSTIDs could be caused by gravity waves originating from the sunset terminator because they have wavefronts almost parallel to the sunset terminator.
\end{abstract}

Key words: MSTID, mid-latitude ionosphere, GPS, total electron contents.

\section{Introduction}

Traveling ionospheric disturbances (TIDs) have been studied for more than four decades since Hines' pioneering work (Hines, 1960) and have been observed using several techniques, such as ionosondes (e.g., Morgan et al., 1978), HF Doppler sounding (e.g., Waldock and Jones, 1986, 1987), satellite beacons (e.g., Evans et al., 1983; Jacobson et al., 1995), and incoherent scatter radars (e.g., Fukao et al., 1991; Kirchengast et al., 1996). TIDs have been thought to be plasma manifestations of atmospheric gravity waves propagating in the thermosphere (Hines, 1960; Hooke, 1968). Many investigations of gravity waves have been carried out because they play an important role in the dynamics of the Earth's atmosphere.

Hunsucker (1982) categorized TIDs with horizontal wavelengths of 100-500 km and periods of $\sim 60$ min into medium-scale TIDs (MSTIDs). Recent observations of airglow images using highly sensitive cooled-CCD cameras have revealed propagation features of nighttime MSTIDs (e.g., Mendillo et al., 1997; Kubota et al., 2000). Most nighttime MSTIDs propagate southwestward (Garcia et al., 2000; Shiokawa et al., 2003a). This preferred propagation direction, however, cannot be explained by the classical theory of gravity waves (Miller et al., 1997; Kelley and Miller, 1997). An electrical force related to electric field perturbation, which could be caused by a plasma instability, is an-

Copyright (c) The Society of Geomagnetism and Earth, Planetary and Space Sciences (SGEPSS); The Seismological Society of Japan; The Volcanological Society of Japan; The Geodetic Society of Japan; The Japanese Society for Planetary Sciences; TERRAPUB other candidate for the source of MSTIDs (Perkins, 1973; Saito et al., 1998; Kelley and Makela, 2001; Shiokawa et al., 2003b). Using satellite beacon data, Jacobson et al. (1995) reported that the propagation direction of MSTIDs is different between daytime and nighttime. Daytime MSTIDs propagate mainly southward, and nighttime MSTIDs propagate southwestward. Miller et al. (1997) suggested that these differences could be due to the different mechanisms producing MSTIDs between daytime and nighttime.

Since the latter half of the 1990s, the Global Positioning System (GPS) has been used to measure total electron content (TEC) along a ray path between the satellite and the receiver. Saito et al. (1998) first showed two-dimensional maps of TEC perturbations caused by MSTIDs over Japan using a dense GPS network which consists of about 1,000 GPS receivers. In Southern California, a large number of GPS receivers have been installed. Using these GPS receivers, Calais et al. (2003) investigated TEC perturbations with 3- to 5-min periods to study infrasonic waves. Moreover, Afraimovich et al. (2004) and Hawarey (2006) investigated TEC perturbations caused by MSTIDs. However, an investigation using the two-dimensional maps of TEC perturbation over Southern California GPS networks has not been carried out.

The present paper shows for the first time twodimensional maps of TEC perturbations caused by MSTIDs over Southern California. Statistical results of large-scale TIDs (LSTIDs) using two-dimensional maps of TEC perturbation obtained from a GPS network in Japan has been reported by Tsugawa et al. (2004), but the present paper re- 


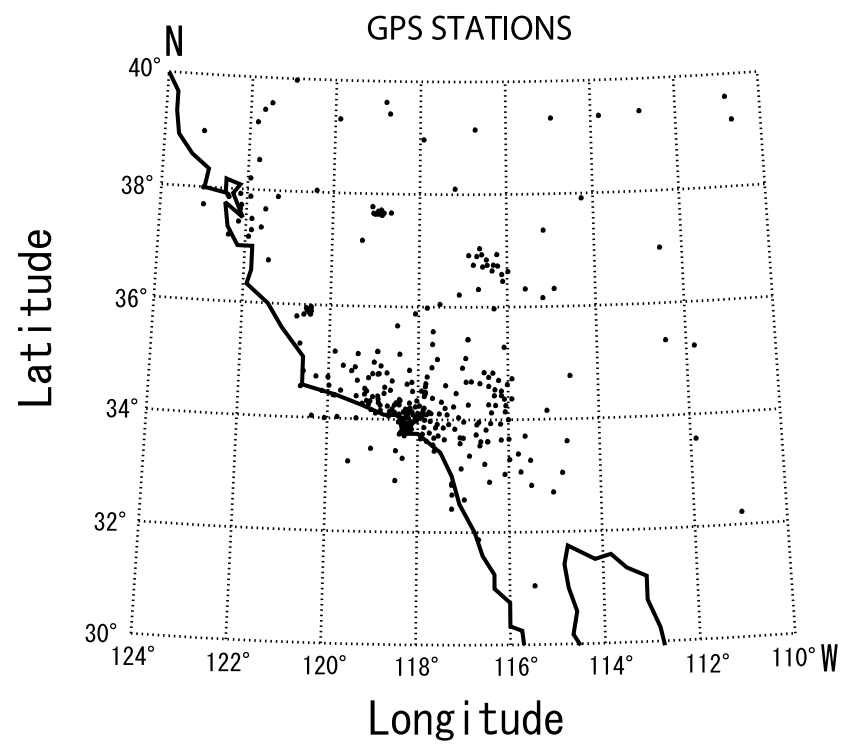

Fig. 1. Locations of GPS receivers in Southern California.

ports the first statistical results of MSTIDs using GPS data obtained in Southern California.

We found that characteristics of MSTIDs are different between daytime, dusk, and nighttime. The possible mechanisms generating MSTIDs are discussed on the basis of these results.

\section{Data and Method of Analysis}

An international GNSS service (IGS), Southern California Integrated GPS Network (SCIGN), and Continuously Operating Reference Stations (CORS) provide RINEX files obtained with dual-frequency GPS receivers throughout the world, in Southern California, and in the USA. Figure 1 shows the locations of the GPS receivers whose data are used in this study. The number of GPS receivers is 365 , and the average distance between two receivers is about $45 \mathrm{~km}$.

GPS data include carrier-phase and group delays (P-code pseudoranges) of dual-frequency $\left(f_{1}=1.57542\right.$ and $f_{2}=$ $1.22760 \mathrm{GHz}$ ) GPS signals every $30 \mathrm{~s}$. TEC along a ray path from the GPS satellite to the receiver is precisely obtained from carrier-phase delays. Slant TEC, $I_{s}$, measured along each GPS satellite-receiver path, can be described by the following equation (Mannucci et al., 1999):

$$
\begin{aligned}
I_{s}= & \frac{1}{40.3} \frac{f_{1}^{2} f_{2}^{2}}{f_{1}^{2}-f_{2}^{2}} \\
& \times\left[\left(L_{1}-L_{2}\right)-\left(\lambda_{1} n_{1}-\lambda_{2} n_{2}\right)+b_{r}+b_{s}\right]
\end{aligned}
$$

where $L_{1}$ and $L_{2}$ are the recorded carrier phases of the signal (converted to distance units), $\lambda_{1}$ and $\lambda_{2}$ are wavelengths of the radio wave, $n_{1}$ and $n_{2}$ are integer cycle ambiguities, and $b_{r}$ and $b_{s}$ are satellite and receiver instrumental biases terms. Because of the ambiguity in the phase measurements, the level of the TEC is unknown. The level is adjusted to that of the TEC derived from the pseudoranges for each satellite-receiver pair. TEC obtained by the above procedure still contains biases inherent in satellite and receiver hardware. To obtain absolute TEC, these biases must be removed. However, we are not interested in the abso- lute TEC, but rather in the perturbation components of TEC $\left(I^{\prime}\right)$. We obtained the $I^{\prime}$ by subtracting the 1-h running average from the time sequence of $I_{s}$. To convert the perturbation of the slant TEC to that of the vertical TEC, $I^{\prime}$ is multiplied by the slant factor. The slant factor is defined as $\tau_{0} / \tau_{1}$, where $\tau_{1}$ is the length of the ray path between 250 and $450 \mathrm{~km}$ altitudes, and $\tau_{0}$ is the thickness of the ionosphere $(200 \mathrm{~km})$ for the zenith path. $I^{\prime}$ obtained from this method clearly represents TEC perturbations caused by MSTIDs. Two-dimensional maps of the TEC perturbations within the area of $110^{\circ}-124^{\circ} \mathrm{W}$ and $30^{\circ}-40^{\circ} \mathrm{N}$ were derived with a time resolution of $30 \mathrm{~s}$ and a spatial resolution of $0.15^{\circ} \times 0.15^{\circ}$ in longitude and latitude. These methods for deriving the two-dimensional TEC perturbation maps were described by Saito et al. (1998). In this study, the TEC data were averaged with their nearby eight grids to compensate for the scarcity of the TEC data distribution. Data derived from a satellite zenith angle smaller than $55^{\circ}$ are used in this study because the data derived from paths with a low elevation angle contain errors associated with cycle slips and slant factor.

We investigated time sequences of two-dimensional TEC perturbation maps to identify the TEC perturbations as MSTIDs. We determined a direction of wavefront of the TEC perturbations by visual assessment of the map of TEC perturbations at every $10 \mathrm{~min}$. Figure 2 shows an example of two-dimensional maps of TEC perturbations during the passage of MSTIDs over Southern California on December 3, 2002. MSTIDs with wavefronts elongated from the northeast to the southwest were observed propagating southeastward between 0830 LT and 0930 LT. A solid line in the maps of Fig. 2 is perpendicular to the wavefront of the TEC perturbations. We assumed that the propagation direction of MSTIDs is perpendicular to the wavefront of the TEC perturbations. To estimate horizontal wavelength and period of MSTIDs, temporal variations of the TEC perturbation along a line parallel to the MSTID propagation direction were investigated. The length of the line was defined as being more than two wavelength. Figure 3 shows temporal variations of the TEC perturbation along the line shown in Fig. 2. The black and white lines in the figure indicate local maxima and minima of the TEC perturbation, respectively. The horizontal wavelength of MSTIDs is defined as twice the distance between the minimum and maximum of the TEC perturbations. The period of the MSTIDs is estimated from the time interval between the minimum and maximum of the TEC perturbations. The horizontal phase velocity $(V)$ of the MSTIDs is obtained from $V=\lambda_{h} / T$, where $\lambda_{h}$ is the horizontal wavelength and $T$ is the period of the MSTIDs.

In this study, MSTIDs are defined as the TEC perturbations which satisfy the following criteria: (1) The TEC perturbation has an amplitude exceeding 0.2 TECU $\left(1 \mathrm{TECU}=10^{16}\right.$ electrons $\left./ \mathrm{m}^{2}\right)$; (2) the horizontal wavelength of the TEC perturbations is shorter than $1,000 \mathrm{~km}$; (3) the period of the TEC perturbations is less than $60 \mathrm{~min}$; (4) the TEC perturbation has more than two wavefronts and propagates on the maps. 


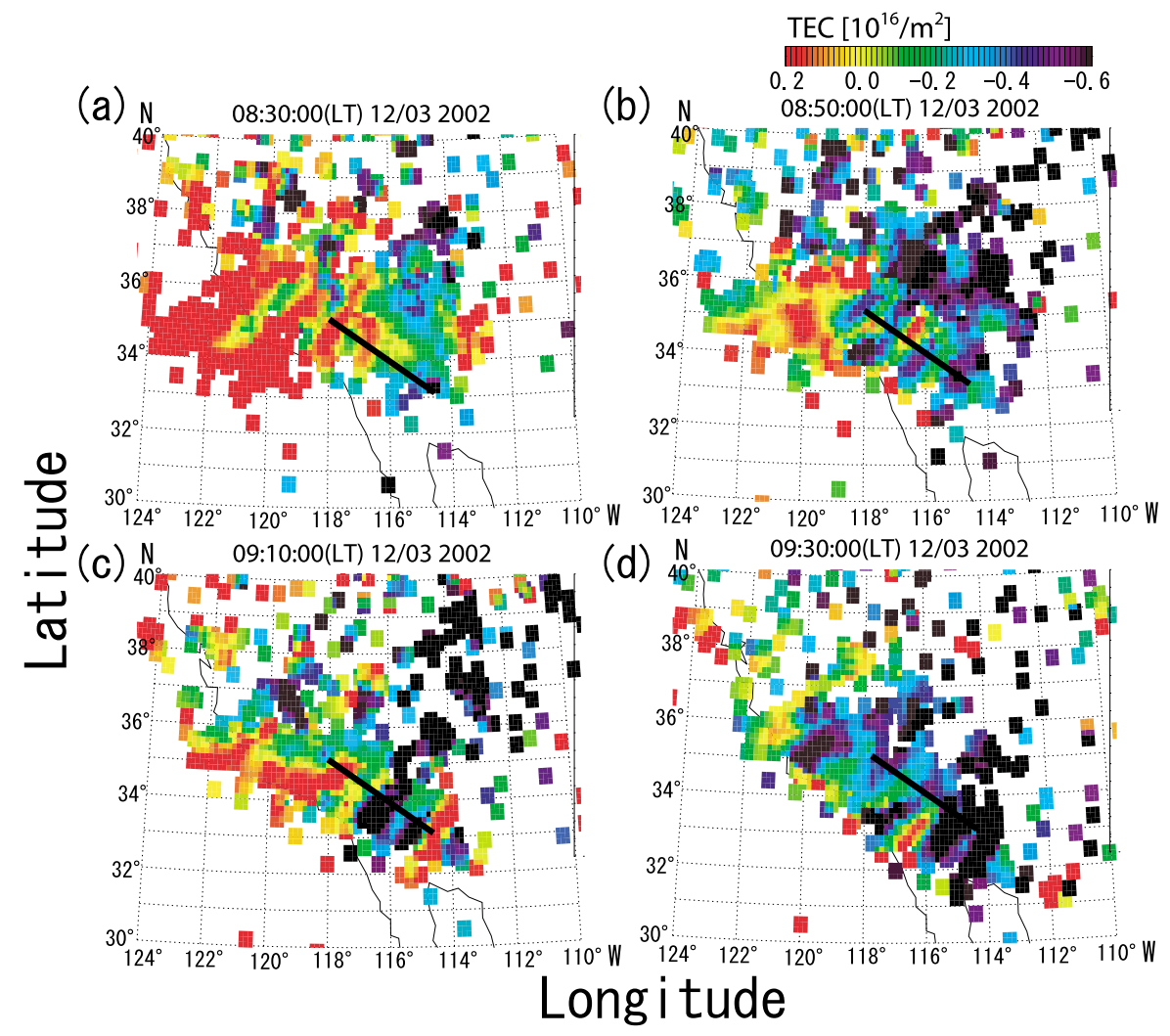

Fig. 2. Two-dimensional maps of TEC perturbations obtained by GPS satellites whose zenith angle is smaller than 55 at 08:30:00-09:30:00 LT on December 3, 2002. The solid line in the maps is perpendicular to the wavefront of MSTIDs.

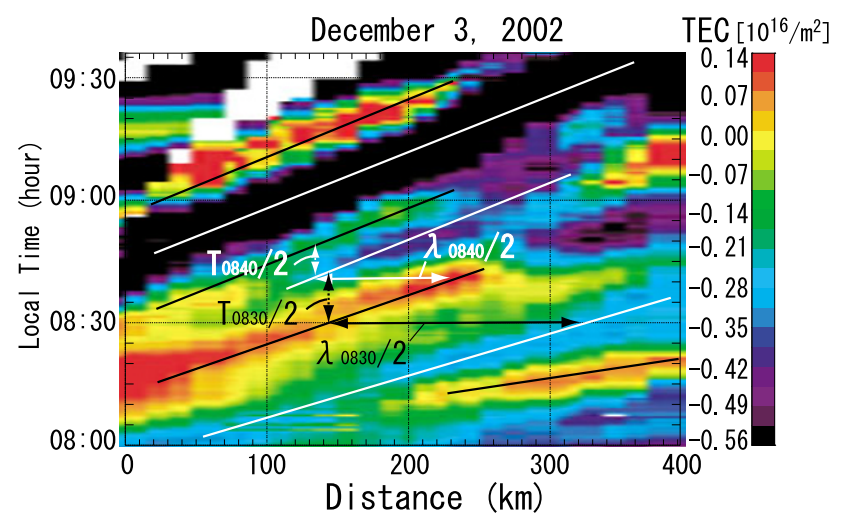

Fig. 3. TEC perturbation along the solid line in Figure 2 between 08:00 and 09:35 LT on December 3, 2002. The black and white lines indicate local maxima and minima of the TEC perturbation, respectively. The length of the solid and break arrow indicates half the period $(T / 2)$, and the half of the horizontal wavelength $(\lambda / 2)$, respectively. The suffix number indicates the local time when $T / 2$ and $\lambda / 2$ of MSTIDs are estimated.

\section{Statistical Results}

We analyzed TEC data obtained in Southern California in 2002 except for 18 days for which data were lacking. To investigate seasonal variations of the characteristics of the MSTIDs, the 1-year interval was divided into three seasons-summer (May-August), winter (November-February), and equinox (March, April, September, and October).

Figure 4 shows local time and seasonal variations of the

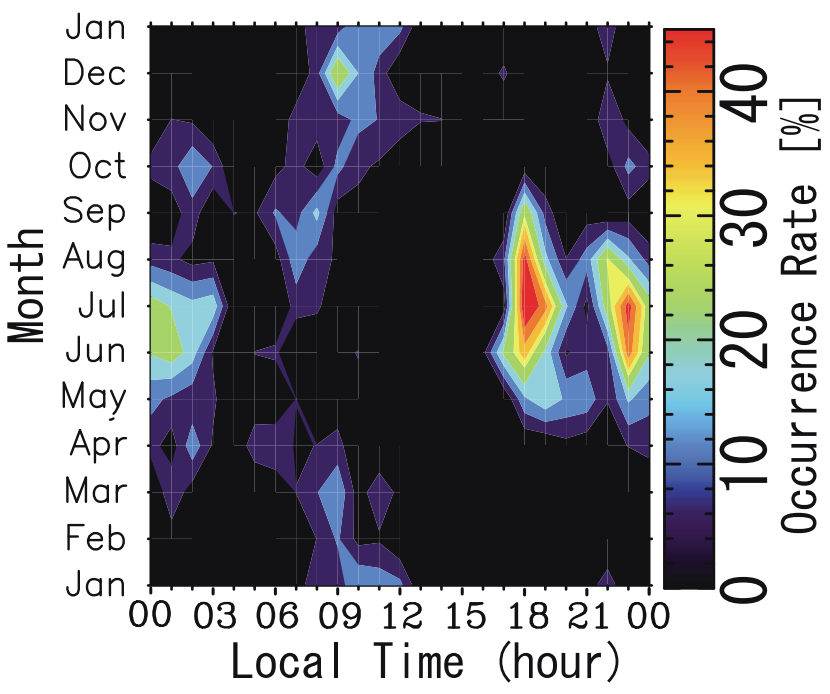

Fig. 4. Local time and seasonal variations of MSTID occurrence rate in Southern California in 2002

MSTID occurrence rate in month-hour bins. The occurrence rate in this figure was defined as the ratio of time period with MSTIDs to monthly and hourly observational intervals. In the figure, the MSTID occurrence rate is high at three regions: morning (0600-1200 LT) in equinoxes and winter, dusk (1700-2000 LT) in summer, and nighttime (2100-0300 LT) in summer. The occurrence rate in the dusk summer is highest and exceeds $40 \%$, which is approximately twice that in morning winter. 


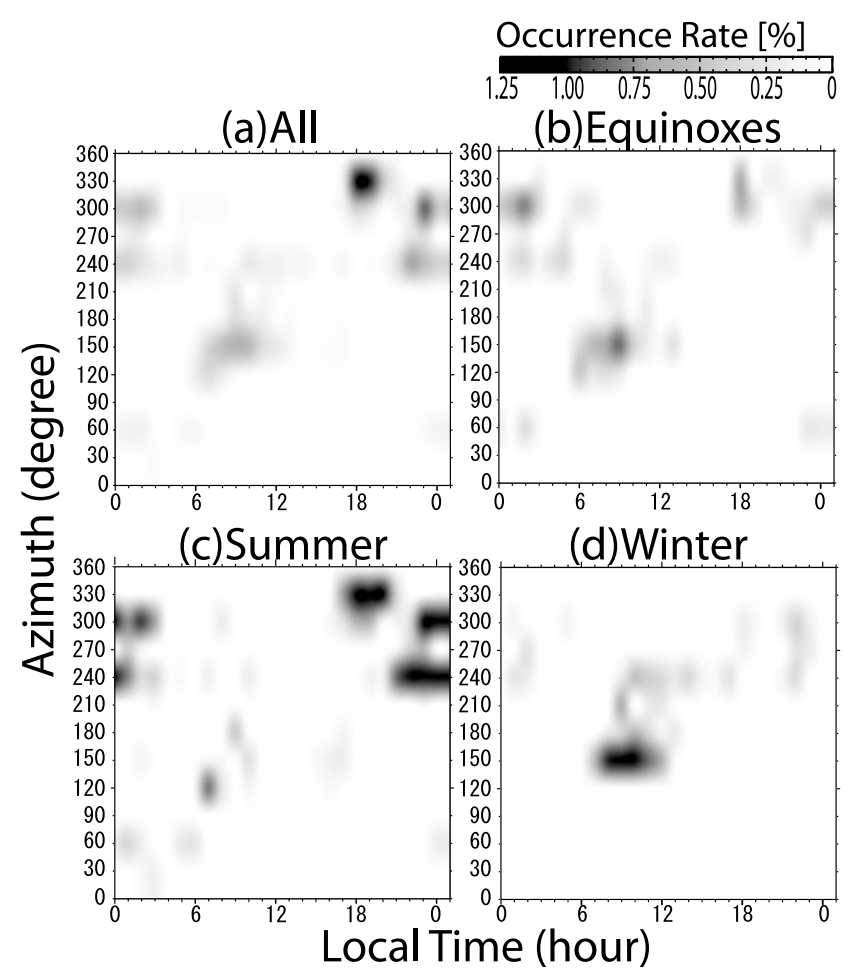

Fig. 5. Local time variation of horizontal propagation direction of MSTIDs for (a) all seasons, (b) equinoxes, (c) summers, and (d) winters.

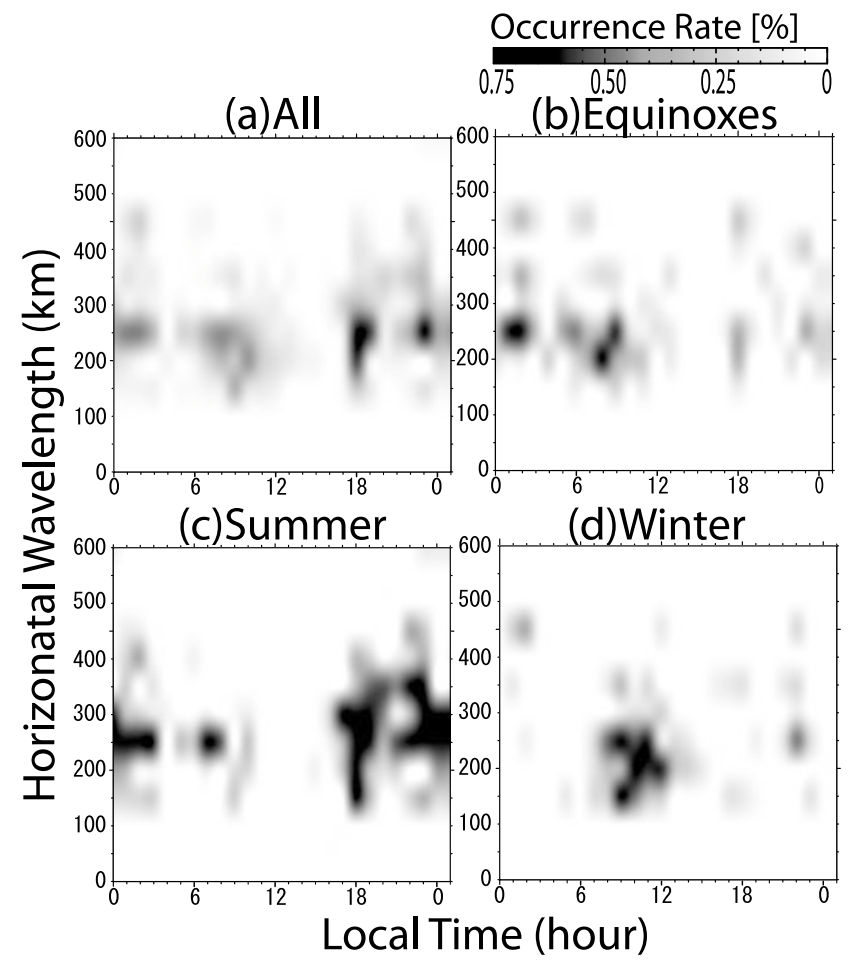

Fig. 6. Local time variation of horizontal wavelength of MSTIDs for (a) all seasons, (b) equinoxes, (c) summers, and (d) winters.

Figure 5 shows the local time variation of the occurrence rate of the MSTID propagation direction for (a) all seasons, (b) equinoxes, (c) summers, and (d) winters. The occurrence rates shown in Figs. 5-8 were defined as the ratio of the time period with MSTIDs to the entire observational in-

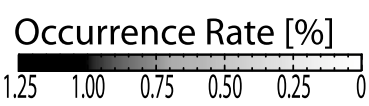

(a)All
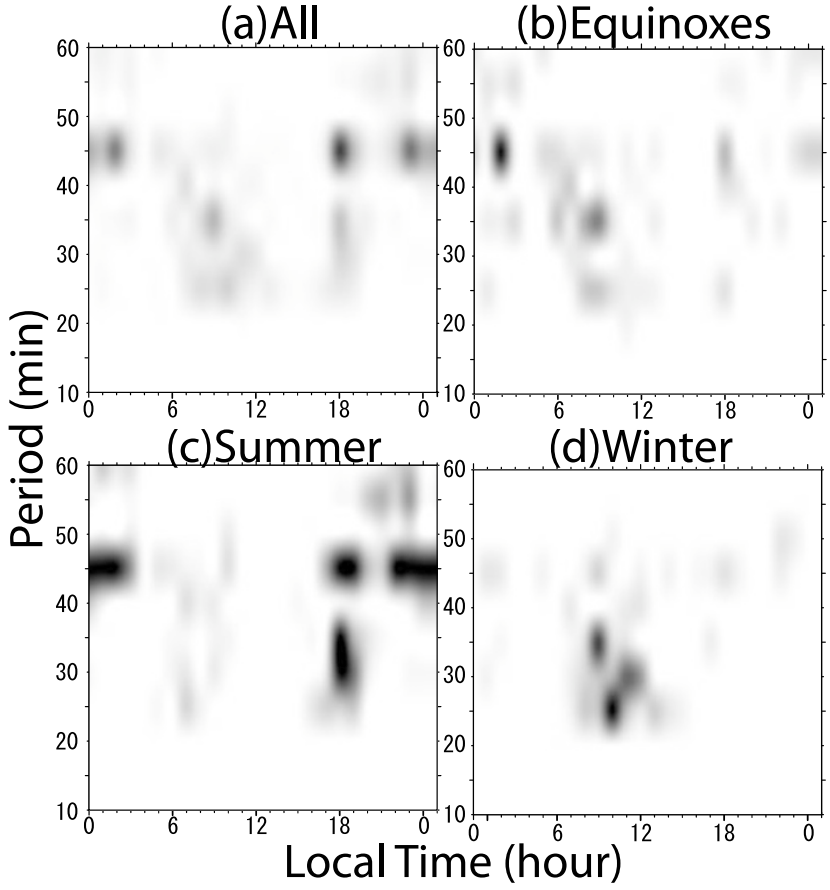

Fig. 7. Local time variation of period of MSTIDs for (a) all seasons, (b) equinoxes, (c) summers, and (d) winters.

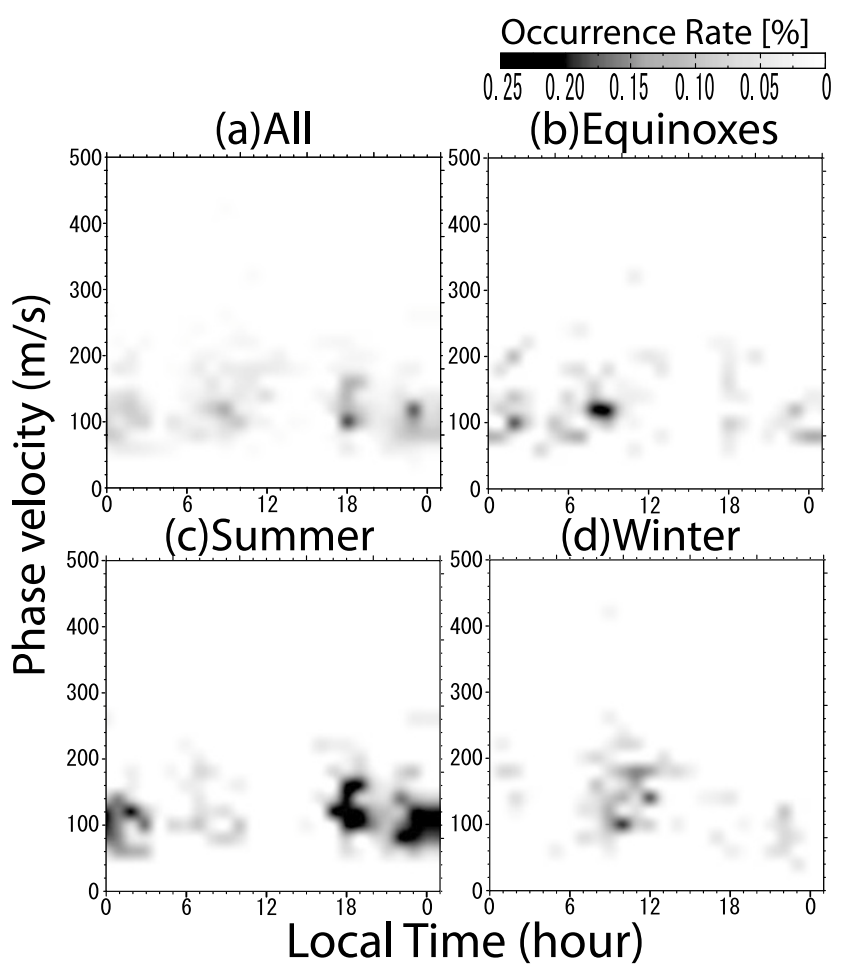

Fig. 8. Local time variation of phase velocity of MSTIDs for (a) all seasons, (b) equinoxes, (c) summers, and (d) winters.

terval. Azimuth was defined as the angle clockwise from the geographical north. All data were classified into $30^{\circ}$ interval bins to calculate the occurrence rate in each azimuthal bin. From the figures, the azimuth of the MSTID propagation direction can be classified into three groups. 
One is a southward propagation observed during daytime as shown in Fig. 5(b) and (d). The propagation direction of the daytime MSTIDs tends to rotate clockwise from $90^{\circ}$ to $240^{\circ}$ (from $120^{\circ}$ to $240^{\circ}$ ) between 0600 and $1500 \mathrm{LT}$ in equinox (winter). The second is a west-northwestward propagation (between $270^{\circ}$ and $330^{\circ}$ in azimuth), as shown in Fig. 5(c). In the summer dusk, these west-northwestward propagating MSTIDs are frequently observed. The third is a southwestward and westward propagation (between $210^{\circ}$ and $300^{\circ}$ in azimuth) observed in the nighttime. This group of MSTIDs is most frequently observed in summer (Fig. 5(c)).

Figure 6 shows the local time variation of the occurrence rate of the horizontal wavelength for (a) all seasons, (b) equinoxes, (c) summers, and (d) winters. Horizontal wavelengths are divided into $50-\mathrm{km}$ bins. Most of the horizontal wavelengths are between 200 and $350 \mathrm{~km}$ in the summer-nighttime as shown in Fig. 6(c), whereas they are between 100 and $250 \mathrm{~km}$ in the winter daytime, as shown in Fig. 6(d). On the other hand, the wavelengths at dusk are widely distributed from 100 to $300 \mathrm{~km}$, as shown in Fig. 6(b).

Figure 7 shows the local time variation in the occurrence rate of period for the MSTIDs for (a) all seasons, (b) equinoxes, (c) summers, and (d) winters. All of the period data were divided into 5-min interval bins. A high occurrence rate can be seen between 40 and $45 \mathrm{~min}$ in the summer nighttime, as shown in Fig. 7(c), and mainly between 20 and $35 \mathrm{~min}$ in the winter daytime, as shown in Fig. 7(d). The period tends to be longer in nighttime than in daytime. On the other hand, the period of the MSTIDs at dusk is widely distributed between 25 and $45 \mathrm{~min}$, as shown in Fig. 7(c).

Figure 8 shows the local time variation in the occurrence rate of horizontal phase velocity for (a) all seasons, (b) equinoxes, (c) summers, and (d) winters. The data were divided into $20-\mathrm{m} / \mathrm{s}$ bin. The phase velocity data are distributed between 60 and $120 \mathrm{~m} / \mathrm{s}$ in the summer nighttime and between 80 and $180 \mathrm{~m} / \mathrm{s}$ in the winter daytime. From a comparison between Fig. 8(c) and (d), the phase velocity is found to be slightly faster in daytime than in nighttime. The phase velocity of the MSTIDs at dusk is widely distributed between 80 and $160 \mathrm{~m} / \mathrm{s}$, as shown in Fig. 8(c).

\section{Discussion}

Using GPS, we have statistically investigated TEC perturbations within a data area of $110^{\circ}-124^{\circ} \mathrm{W}$ and $30^{\circ}-40^{\circ} \mathrm{N}$ obtained in Southern California to reveal seasonal variation of the MSTID occurrence rate, propagation direction, period, horizontal wavelength, and phase velocity. From the characteristics of the observed MSTIDs, we can categorize the MSTIDs into the three following types: (1) daytime MSTIDs which propagate southeastward and frequently occur in the winter morning and equinoxes; (2) nighttime MSTIDs which propagate southwestward and frequently occur in the summer; (3) dusk MSTIDs which propagate northwestward and frequently occur in summer.

Using TEC data obtained from VHF radio beacons from two geosynchronous satellites, Jacobson et al. (1995) reported that the daytime and nighttime MSTIDs over Los Alamos $\left(35.9^{\circ} \mathrm{N}, 106.3^{\circ} \mathrm{W}\right)$ have different seasonal varia- tions in terms of their occurrence rate and their propagation direction. They showed that the daytime MSTIDs often occur during winter and equinoxes, at which time they propagate southward, while the nighttime MSTIDs often occur during summer solstice through autumn equinox and propagate toward the west/northwest. Using two-dimensional radio-interferometic observations of cosmic radio-sources obtained by the Nancay Radiohelograph (NRH) located at Nancay $\left(47.3^{\circ} \mathrm{N}, 2.2^{\circ} \mathrm{E}\right)$ in France between 1988 and 1990, Mercier (1996) showed the bimodal time distribution of TIDs with preferential directions towards the southeast during the daytime and toward the southwest during the nighttime. They also described that the nighttime TIDs have a longer period than daytime TIDs. These results are mostly consistent with those found in the present study. Kelley and Miller (1997) and Kotake et al. (2006) have pointed out that the difference between daytime and nighttime MSTIDs in terms of their propagation direction would be responsible for the difference in mechanisms causing MSTIDs. In the following subsections, we discuss the mechanisms causing the MSTIDs in the daytime, nighttime and dusk.

\subsection{Daytime MSTIDs}

As shown in Section 3, daytime MSTIDs occur frequently in the winter and during equinoxes and have a wavelength of $100-250 \mathrm{~km}$. The propagation direction of the daytime MSTIDs is mostly southeastward and rotates from southeastward to southwestward with time. Using satellite tracking radar at Millstone Hill $\left(42.6^{\circ} \mathrm{N}, 71.5^{\circ} \mathrm{W}\right)$, Evans et al. (1983) reported that the daytime MSTIDs occurrence rate is high in winter and that the horizontal wavelength is $200-250 \mathrm{~km}$. These results are consistent with our results. Using the Middle and Upper atmosphere (MU) radar installed at Shigaraki $\left(34.8^{\circ} \mathrm{N}, 136.1^{\circ} \mathrm{E}\right)$, Japan, Oliver et al. (1997) reported a preference for the southward propagation of the daytime MSTIDs with periods of 40-130 min. This preference is also consistent with our results.

MSTIDs have been thought to be caused by atmospheric gravity waves (Hines, 1960; Hooke, 1968). Ions in the $F$ region move along the geomagnetic field lines through the neutral-ion collisions. Hooke (1968) has shown that the velocity of the ion motion along the geomagnetic field is the same as that of neutral motion along the geomagnetic field caused by gravity waves. However, the ion motion across the magnetic field line $(\mathbf{B})$ is restricted because the ion gyro-frequency is much higher than the ion-neutral collision frequency. This directivity of the ion mobility causes directivity in the response of the electron density variations to the neutral motion due to the gravity waves. Since neutral particle oscillation parallel to $\mathbf{B}$ is larger for gravity waves propagating equatorward than for those propagating to other directions, equatorward-propagating gravity waves could cause a larger amplitude of TEC perturbations than gravity waves propagating in other directions (Hooke, 1970). Such directivity in response to the $F$ region plasma of gravity waves could be responsible for the southward preference in the propagation directions of the daytime MSTIDs.

The motion of the neutral particles across the magnetic field line results in an anisotropic frictional ion drag force (Hines, 1968; Liu and Yeh, 1969; Kelley and Miller, 1997). 
The ion drag force dumps neutral particle oscillation due to the gravity waves. Liu and Yeh (1969) derived the general dispersion relation of gravity waves, including the constant collision frequency, and studied the effects of the ion drag on their propagation. Kelley and Miller (1997) showed that a minimum of ion drag force occurs for waves propagating equatorward. Such ion drag force also contributes to the southward preference in the propagation directions of the observed daytime MSTIDs.

As shown in Fig. 5(b) and (d), the propagation direction of the daytime MSTIDs tends to rotate clockwise from $90^{\circ}$ to $240^{\circ}\left(120^{\circ}\right.$ to $\left.240^{\circ}\right)$ in azimuth between 0600 and 1500 LT in equinox (winter). This feature is consistent with that reported in earlier publications. Kalikhman (1980) showed local time variation of MSTID propagation direction. He observed MSTIDs in Irkutsk $\left(52^{\circ} \mathrm{N}, 104^{\circ} \mathrm{E}\right)$, Russia during 1975-1976. His measurements were performed during the daytime using Doppler and arrival angle data obtained from vertical sounding of the $F_{2}$-layer. His results showed that the MSTID propagation directions were 90$150^{\circ}, 120-180^{\circ}$, and $150-210^{\circ}$ in azimuth at 8-11, 1114, and 14-17 LT, respectively, which is rotating clockwise with time. Afraimovich et al. (1999) presented diurnal and seasonal variations of MSTIDs, using data from an annual run of ETS 2 radio signal polarization, angle-of-arrival, and scintillation measurements at $136 \mathrm{MHz}$ in Irkutsk $\left(52^{\circ} \mathrm{N}\right.$, $\left.104^{\circ} \mathrm{E}\right)$, Russia. The MSTID propagation direction of daytime MSTIDs is $160^{\circ}$ in azimuth, on average, and rotates clockwise with time. Furthermore, they pointed out that the propagation direction is opposite to the direction of the neutral wind in the thermosphere. These local time variations of MSTID propagation direction could be explained by the wind filtering mechanism of gravity waves (Cowling et al., 1971; Waldock and Jones, 1984). When the horizontal phase velocity of the gravity waves is close to the background neutral wind velocity (a critical level), the gravity waves can not propagate through the critical level (Bretherton, 1967; Kalikhman, 1980). The favored azimuth of the MSTIDs propagation could counter the background wind. The background wind in the thermosphere is predominantly a diurnal tide with a clockwise rotation and northward direction at local noon. Consequently, the preferred azimuth of the MSTIDs propagation direction could be southward at around local noon, rotating clockwise with time.

Afraimovich et al. (2003) identified a specific class of mid-latitude MSTIDs, namely traveling wave packets (TWPs), which consisted of quasi-monochromatic oscillations of TEC with a period of around 10-20 min, and reported morphology of TWPs using the global GPS network data obtained at California, the Caribbean basin, and SouthEast Asia for 105 days in 1998-2001. Most of the TWPs were observed during daytime winter, and their propagation direction and propagation velocity is southward $\left(146^{\circ}\right.$ in azimuth) and more than $180 \mathrm{~m} / \mathrm{s}$, respectively, on average. These parameters are consistent with those of the daytime MSTIDs reported in this study, whereas typical periods of TWPs are shorter than those of MSTIDs observed in this study.

\subsection{Nighttime MSTIDs}

Our statistical results have showed that most nighttime MSTIDs propagate southwestward. This southwestward preference of nighttime MSTID propagation is consistent with that obtained from previous observations using a 630nm airglow imaging technique (e.g., Miller et al., 1997; Garcia et al., 2000; Kelley and Makela, 2001; Shiokawa et al., 2003a). Garcia et al. (2000) and Shiokawa et al. (2003a) investigated statistically the period, phase velocity, and horizontal wavelength of nighttime MSTIDs. Using 630$\mathrm{nm}$ emission data taken from Rikubetsu $\left(43.5^{\circ} \mathrm{N}, 143.8^{\circ} \mathrm{E}\right)$ and Shigaraki $\left(34.8^{\circ} \mathrm{N}, 136.1^{\circ} \mathrm{E}\right)$, Japan, Shiokawa et al. (2003a) reported that the horizontal wavelength, period, and phase velocity are $100-300 \mathrm{~km}, 0.5-1.5$ hour, and 50-100 $\mathrm{m} / \mathrm{s}$, respectively. Using 630-nm emission data obtained from Arecibo $\left(18.3^{\circ} \mathrm{N}, 66.7^{\circ} \mathrm{W}\right)$, Pueruto Rico, Garcia et al. (2000) also reported that the horizontal wavelength, period, and phase velocity are $100-250 \mathrm{~km}, 0.5-1.5$ hour, and $60-120 \mathrm{~m} / \mathrm{s}$, respectively. In this study, the horizontal wavelength, period, and phase velocity are $200-250 \mathrm{~km}, 40-45$ min, and 40-120 m/s, respectively. The period obtained in this study is shorter than that obtained from airglow observations. This difference could be caused by the difference in the method of the data processing. In this study, perturbation components of TEC are derived from subtracting 1-h running average, so that a period of more than $1 \mathrm{~h}$ cannot be detected. The horizontal wavelength and phase velocity obtained in this study are consistent with those obtained in the above previous studies.

We briefly review nighttime MSTIDs below. When spatial variation of the plasma density in the $F$ region exists, polarized electric fields could be generated by the ionospheric electric current to maintain continuity of the electric current. Although such polarized electric fields generated in the $F$ region are short-circuited by high conductivity in the $E$ region in daytime, polarized electric fields are maintained in nighttime because the $E$-region conductivity is significantly reduced due to recombination of the $E$ region plasma. Field-line-integrated electric current in the $F$ region, $\mathbf{J}$, is written as

$$
\mathbf{J}=\Sigma_{P}^{F}(\mathbf{E}+\mathbf{U} \times \mathbf{B})
$$

where $\Sigma_{P}^{F}$ is the field-line-integrated conductivity, $\mathbf{E}$ is the background electric field, $\mathbf{U}$ is the neutral wind. During nighttime, $\mathbf{E}$ is expected to be smaller than-and antiparallel to $-\mathbf{U} \times \mathbf{B}$ because $\mathbf{E}$ is generally generated by the $F$-region dynamo mechanism (Rishbeth, 1971). Consequently, $\mathbf{J}$ probably flows to almost the same direction as $\mathbf{U} \times \mathbf{B}$. Since $\mathbf{U}$ flows southeastward during nighttime (HWM model; Hedin et al., 1996), J could flow northeastward. In the condition that $\mathbf{J}$ traverses the perturbations of $\Sigma_{P}^{F}$ (wavefront of MSTIDs), polarized electric fields $\left(\mathbf{E}_{\mathrm{p}}\right)$ should be generated to maintain divergence-free ionospheric currents. Therefore, $\mathbf{E}_{\mathrm{p}}$ is perpendicular to the wavefronts of MSTIDs, and northeastward (southwestward) in the regions of low (high) $\Sigma_{P}^{F}$. $\mathbf{E}_{\mathrm{p}}$ moves the plasma upward (downward) by $\mathbf{E} \times \mathbf{B}$ drift to cause the plasma density perturbations. Actually, Shiokawa et al. (2003b) have shown that electric field perturbations, measured by satellite, are associated with MSTID structures in airglow im- 
ages and that the direction of the electric fields is consistent with that of the polarized electric fields which are expected to be generated by the spatial perturbations of $\Sigma_{P}^{F}$. When the direction of the MSTID wave vector $\mathbf{k}$ is between the direction of the effective electric field $(\mathbf{E}+\mathbf{U} \times \mathbf{B})$ and the geomagnetic east, the perturbations in the polarized electric fields and plasma density grow with time by the Perkins instability (Perkins, 1973; Hamza, 1999). Alignment of the observed MSTIDs wavefront (from northwest to southeast) is consistent with that predicted by the Perkins instability. However, the observed southwestward propagation is opposite to the direction predicted by the Perkins instability because the structures generated by the Perkins instability should propagate northeastward with the background $\mathbf{E} \times \mathbf{B}$ drift, which is almost always to the east (Garcia et al., 2000). To explain the observed southwestward preference, Kelley and Makela (2001) have proposed a mechanism in which polarized electric fields in the direction along the horizontal wavefront of the MSTIDs are considered, assuming that the MSTID structure is of finite size parallel to the horizontal wavefront.

Our observational results show that the nighttime MSTIDs in Southern California have a major peak in summer. This seasonal variation in the nighttime MSTIDs is consistent with that of the nighttime MSTIDs observed in 630-nm airglow imagers in Japan (Shiokawa et al., 2003a). On the other hand, Garcia et al. (2000) have reported a major peak in the occurrence rate in winter over Arecibo. The major peak of the MSTID occurrence in the summer or winter solstice could be explained by the linear growth rate of the Perkins instability, which is inversely proportional to the neutral density (Perkins, 1973). The neutral density shows semiannual variation with maxima at equinoxes and minima at solstices. Kotake et al. (2006) have shown that the occurrence rate of nighttime MSTIDs has a longitudinal dependence. Bowman (1992) has suggested that these longitudinal variations in the MSTIDs occurrence rate are also responsible for the longitudinal variation of the neutral density.

\subsection{Dusk MSTIDs}

We found that MSTID occurrence reaches a peak at summer dusk. The dusk MSTIDs have wavefronts elongated from northeast to southwest and propagate northwestward. Therefore, the MSTIDs wavefronts are almost parallel to the sunset terminator in summer. This feature is consistent with that of MSTIDs observed at the sunrise and sunset terminators based on incoherent scatter radar measurements at Millstone Hill $\left(42.6^{\circ} \mathrm{N}, 71.5^{\circ} \mathrm{W}\right)$ (Galushko et al., 1998). Based on characteristics of the observed MSTIDs, these researchers suggested that the observed MSTIDs could be caused by gravity waves generated by the solar terminator. They also reported that the most powerful spectral components of the electron density fluctuations caused by MSTIDs were concentrated within periods of 1.5-2.5 h, although the spectral components spread in other periods. Periods of the MSTIDs observed in this study are within a range of 20 to $45 \mathrm{~min}$. This difference could be caused by the method of the data processing. In this study, perturbation components of TEC are derived from subtracting a 1-h running average.

\section{Conclusion}

We have shown for the first time statistical characteristics of the MSTIDs over Southern California observed with densely spaced GPS receivers, and found that characteristics of MSTIDs are different at daytime, dusk, and nighttime, respectively. The observed characteristics can be summarized as follows:

1. The occurrence rate of daytime MSTIDs is high in winter and during equinoxes. The horizontal wavelength, period, and phase velocity of daytime MSTIDs are 100-250 $\mathrm{km}, 20-35 \mathrm{~min}$, and $80-180 \mathrm{~m} / \mathrm{s}$, respectively. Most of the daytime MSTIDs propagate southeastward. Since neutral particle oscillation parallel to the geomagnetic field line is larger for gravity waves propagating equatorward than for gravity waves propagating in other directions, equatorwardpropagating gravity waves could cause a larger amplitude of TEC perturbations than gravity waves propagating toward other directions. Such directivity in response to the $F$ region plasma to the gravity waves could be responsible for the southward preference in the propagation directions of the daytime MSTIDs. Dumping of the gravity waves due to the ion drag also could contribute to this directivity.

2. The occurrence rate of nighttime MSTIDs has a peak in summer. The propagation direction, horizontal wavelength, period, and phase velocity are southwest, 200-350 $\mathrm{km}, 40-45 \mathrm{~min}$, and $60-120 \mathrm{~m} / \mathrm{s}$, respectively. These features are consistent with previous MSTID statistics obtained from 630-nm airglow imaging observations in Japan and Puerto Rico. From the propagation direction of the nighttime MSTIDs, electrodynamical forces, such as the Perkins instability, could play an important role in generating the nighttime MSTIDs.

3. The occurrence rate of dusk MSTIDs is high in summer. Their horizontal wavelength, period, and phase velocity are $100-300 \mathrm{~km}, 20-45 \mathrm{~min}$, and $80-160 \mathrm{~m} / \mathrm{s}$, respectively. They propagate northwestward. Because dusk MSTIDs have wavefronts elongated from the northeast to the southwest, they could be caused by gravity waves generated at the solar terminator.

The characteristics of MSTIDs obtained in this study are consistent with previous observational results obtained previously. These results suggest that mechanisms for generating MSTIDs are different between daytime, nighttime and dusk, respectively.

Acknowledgments. GPS data were supplied by the International GNSS Service (IGS), Southern California Integrated GPS Network (SCIGN) and Continuously Operating Reference Stations (CORS). This work is supported by a Grant-in-Aid for Scientific Research (18654083) of the Ministry of Education, Culture, Sports, Science and Technology of Japan, and partly by the 21 st Century COE Program "Dynamics of the Sun-Earth-Life Interactive System (SELIS)" of Nagoya University, Japan. N. Kotake is partly supported by the 21st Century COE Program "Information Nano-Devices Based on Advanced Plasma Science" of Nagoya University, Japan.

\section{References}

Afraimovich, E. L., O. N. Boitman, E. I. Zhovty, A. D. Kalikhman, and T. G. Porog, Dynamics and anisotropy of traveling ionospheric disturbances as deduced from transionospheric sounding data, Radio. Sci., 34, 477-487, 1999.

Afraimovich, E. L., N. P. Perevalova, and S. V. Voyeikov, Traveling wave 
packets of total electron content disturbances as deduced from global GPS network data, J. Atmos. Solar-Terr. Phys., 65, 1245-1262, 2003.

Afraimovich, E. L., E. I. Astafieva, E. I. Astafieva, M. B. Gokhberg, V. M. Lapshin, V. E. Permyakova, G. M. Steblov, and S. L. Shalimov, Variations of the total electron content in the ionosphere from GPS data recorded during the Hector Mine earthquake of October 16, 1999, California. Russ. J. Earth Sci., 6, 339-354, 2004.

Bowman, G. G., Upper atmosphere neutral-particle density variations compared with spread-F occurrence rates at locations around the world, Ann. Geophys., 10, 676-682, 1992.

Bretherton, F. P., The propagation of groups of gravity waves in a shear flow, Quant. J. R. Meteorol. Soc., 92, 466-480, 1967.

Calais, E., J. S. Haase, and J. B. Minster, Detection of ionospheric perturbations using a dense GPS array in Southern California, Geophys. Res. Lett., 30, 1-4, 2003.

Cowling, D. H., H. D. Webb, and K. C. Yeh, Group rays of internal gravity waves in a wind stratified atmosphere, J. Geophys. Res., 76, 213-220, 1971.

Evans, J. V., J. M. Holt, and R. H. Wand, A differential-Doppler study of traveling ionospheric disturbances from Millstone Hill, Radio Sci., 18, 435-451, 1983.

Fukao, S., Y. Yamamoto, W. L. Oliver, T. Takami, M. D. Yamanaka, M. Yamamoto, T. Nakamura, and T. Tsuda, Middle and upper atmosphere radar observations of ionospheric horizontal gradients produced by gravity waves, J. Geophys. Res., 98, 9443-9451, 1991.

Garcia, F. J., M. C. Kelley, J. J. Makela, and C. S. Huang, Airglow observations of mesoscale low-velocity traveling traveling ionospheric disturbances at midlatitudes, J. Geophys. Res., 105, 18,407-18,415, 2000.

Galushko, V. G., V. V. Paznukhov, Y. M. Yampolski, and J. C. Foster, Incoherent scatter radar observations of AGW/TID events generated by the moving solar terminator, Ann. Geophys., 16, 821-827, 1998.

Hamza, A. M., Perkins instability revisited, J. Geophys. Res., 104, 22,56722,575, 1999.

Hawarey, M., Traveling ionospheric disturbance over California mid 2000, Nonlinear Proc. Geophys., 13, 1-7, 2006.

Hedin, A. E., E. L. Fleming, A. H. Manson, F. J. Scmidlin, S. K. Avery, R. R. Clark, S. J. Franke, G. J. Fraser, T. Tsunda, F. Vial, and R. A. Vincent, Empirical wind model for the upper, middle, and lower atmosphere, $J$. Atmos. Terr. Phys., 58, 1421-1447, 1996.

Hines, C. O., Internal atmospheric gravity waves at ionospheric heights, Can. J. Phys., 38, 1441-1481, 1960.

Hines, C. O., An effect of ohmic losses in upper atmospheric gravity waves, J. Atmos. Terr. Phys., 30, 851-856, 1968.

Hooke, W. H., Ionospheric irregularities produced by internal atmospheric gravity waves, J. Atmos. Terr. Phys., 30, 795-823, 1968.

Hooke, W. H., The ionospheric response to internal gravity waves 1 . The $F$ region response, J. Geophys. Res., 75, 5535-5544, 1970.

Hunsucker, R., Atmospheric gravity waves generated in the highlatitude ionosphere: a review, Rev. Geophys. Space Phys., 20, 293-315, 1982.

Jacobson, A. R., R. C. Carlos, R. S. Massey, and G. Wu, Observations of traveling ionospheric disturbances with a satellite-beacon radio interferometer: Seasonal and local time behavior, J. Geophys. Res., 100, $1653-1665,1995$.

Kalikhman, A. D., Medium-scale traveling ionospheric disturbances and thermospheric winds inthe F-region, J. Atmos. Soler-Terr. Phys., 42, 697-703, 1980.

Kelley, M. C. and J. J. Makela, Resolution of the discrepancy between experiment and theory of midlatitude $F$-region structures, Geophys. Res. Lett., 28, 2589-2592, 2001.

Kelley, M. C. and C. A. Miller, Electrodynamics of midlatitude $\operatorname{spread} F$, 3. Electrohydrodynamic waves? A new look at the role of electric fields in thermospheric wave dynamics, J. Geophys. Res., 102, 11,539-11,547, 1997.

Kirchengast, G., K. Hocke, and K. Schlegel, The gravity wave-TID re- lationship: insight via theoretical model-EISCAT data comparison, $J$. Atmos. Terr. Phys., 58, 233-243, 1996.

Kotake, N., Y. Otsuka, T. Tsugawa, T. Ogawa, and A. Saito, Climatological study of GPS total electron content variations caused by mediumscale traveling ionospheric disturbances, J. Geophys. Res., 111, A04306, doi:10.1029/2005JA011418, 2006.

Kubota, M., K. Shiokawa, M. K. Ejiri, Y. Otsuka, T. Ogawa, T. Sakanoi, H. Fukunishi, M. Yamamoto, S. Fukao, and A. Saito, Traveling ionospheric disturbances observed in the OI 630-nm nightglow images over Japan by using a multi-point imager network during the FRONT campaign, Geophys. Res. Lett., 24, 4037-4040, 2000.

Liu, C. H. and K. C. Yeh, Effect of ion drag on propagation of acousticgravity waves in the atmospheric $F$ region, J. Geophys. Res., 74, 22482255,1969

Mannucci, A. J., B. A. Iijima, U. J. Lindqwister, X. Pi, L. Sparks, and B. D. Wilson, 25. GPS and Ionosphere, in Review of Radio Science 19961999, edited by W. R. Stone, 625-665, URSI, 1999.

Mendillo, M., J. Baumgardner, D. Nottingham, J. Aarons, B. Reinisch, J. Scali, and M. Kelley, Investigations of thermospheric-ionospheric dynamics with 6300- $\AA$ images from the Arecibo Observatory, J. Geophys. Res., 102, 7331-7343, 1997.

Mercier, C., Some characteristics of atmospheric gravity waves observed by radio-interferometry, Ann. Geophys., 14, 42-58, 1996.

Miller, C. A., W. E. Swartz, M. C. Kelley, M. Mendillo, D. Nottingham, J. Scali, and B. Reinisch, Electrodynamics of midlatitude spread $F$, 1. Observations of unstable, gravity wave-induced ionospheric electric fields at tropical latitudes, J. Geophys. Res., 102, 11,521-11,532, 1997.

Morgan, M. G., C. H. J. Calderon, and K. A. Ballard, Techniques for the study of TID's with multi-station rapid-run ionosondes, Radio Sci., 13, 729-741, 1978.

Oliver, W. L., Y. Otsuka, M. Sato, T. Takami, and S. Fukao, A climatology of $F$ region gravity wave propagation over the middle and upper atmosphere radar, J. Geophys. Res., 102, 14,499-14,512, 1997.

Perkins, F., Spread F and ionospheric currents, J. Geophys. Res., 78, 218226, 1973.

Rishbeth, H., The F-layer dynamo, Planet. Space Sci., 19, 263-267, 1971.

Saito, A., S. Miyazaki, and S. Fukao, High resolution mapping of TEC perturbations with the GSI GPS network over Japan, Geophys. Res. Lett., 25, 3079-3082, 1998.

Shiokawa, K., C. Ihara, Y. Otsuka, and T. Ogawa, Statistical study of nighttime medium-scale traveling ionospheric disturbances using midlatitude airglow images, J. Geophys. Res., 108, 1052, doi:10.1029/2002JA009491, 2003a.

Shiokawa, K., C. Ihara, Y. Otsuka, and T. Ogawa, Ground and satellite observations of nighttime medium-scale traveling ionospheric disturbance at midlatitude, J. Geophys. Res., 108, 1145, doi:10.1029/2002JA009639, 2003b.

Tsugawa, T., A. Saito, and Y. Otsuka, A statistical study of large-scale traveling ionospheric disturbances using the GPS network in Japan, $J$. Geophys. Res., 109, 6302, doi:10.1029/2003JA010302, 2004.

Waldock, J. A. and T. B. Jones, The effects of neutral winds on the propagation of medium-scale atmospheric gravity wave at mid-latitudes, $J$. Atmos. Terr. Phys., 46, 217-231, 1984.

Waldock, J. A. and T. B. Jones, HF Doppler observations of medium-scale traveling ionospheric disturbances observed at mid-latitudes, J. Atmos. Terr. Phys., 48, 245-260, 1986.

Waldock, J. A. and T. B. Jones, Source regions of medium scale traveling ionospheric disturbances observed at mid-latitudes, J. Atmos. Terr. Phys., 49, 105-114, 1987.

N. Kotake (e-mail: kotake@stelab.nagoya-u.ac.jp), Y. Otsuka, T. Ogawa, T. Tsugawa, and A. Saito 